\begin{tabular}{ccc}
\hline & International Journal of Engineering \& Technology, $7(2.32)(2018) 228-232$ \\
SPC & International Journal of Engineering \& Technology \\
Website: www.sciencepubco.com/index.php/IJET & Research paper \\
\hline
\end{tabular}

\title{
A Novel Approach on Suggesting Travel Route by Efficient Watchword
}

\author{
Venkata Naresh Mandhala ${ }^{1}$, N.Ganesh ${ }^{2}$, K.Sai Shirini ${ }^{3}$, Ch.Alekhya Sri ${ }^{4}$ \\ ${ }^{1,2,3,4}$ Department of CSE, Koneru Lakshmaiah Education Foundation, Vaddeswaram, India \\ *Corresponding author E-mail: mvnaresh.mca@gmail.com
}

\begin{abstract}
With the fame of web-based social networking (e.g., Facebook ), clients can undoubtedly share their registration records and photographs amid their treks. In perspective of the gigantic number of client verifiable portability records in web-based social networking, we plan to find venture out encounters to encourage trip arranging. When arranging a trek, clients dependably have specific inclinations with respect to their outings. Rather than confining clients to restricted question alternatives, for example, areas, exercises, or eras, we consider discretionary portrayals as watchwords about customized necessities. In addition, an assorted and agent set of suggested travel courses is required. Earlier works have expounded on mining and positioning existing courses from registration information. To address the issue for programmed trip association, we guarantee that more highlights of Spots of Intrigue (POIs) ought to be extricated. Along these lines, in this paper, we propose an efficient Catchphrase mindful Agent Travel Course structure that utilizations learning extraction from clients' verifiable portability records and social associations. Expressly, we have planned a watchword extraction module to group the POI-related labels, for successful coordinating with question catchphrases. We have additionally planned a course recreation calculation to develop course hopefuls that fulfill the necessities. To give befitting question comes about, we investigate Agent Horizon ideas, that is, the Horizon courses which best depict the exchange offs among various POI highlights. To assess the viability and efficiency of the proposed calculations, we have led broad investigates genuine area based informal community datasets, and the examination comes about demonstrate that our strategies do in fact illustrate great execution contrasted with best in class works.
\end{abstract}

Keywords:Spatial keyword query, LBSN, Spatial database, POI, Watchword..

\section{Introduction}

Data mining is the methods for removing information from a dataset for clients to utilize it in different purposes. The motivation behind such information assumes a noteworthy part in catchphrase seeking. Seeking is a typical action occurring in information mining. Scanning for spatial articles from spatial database has as of late started excitement among scientists. This roused to create strategies to recover spatial articles. Spatial items comprise of articles related with spatial highlights. As it were, spatial items include spatial information alongside longitude and scope of area. Questioning such information is called best catchphrase cover questioning. Hunt is called best catchphrase cover look. Existing strategy to such information consider either least entomb target separation and catchphrase seek. Accordingly new techniques for best catchphrase cover seek was created. Customary closest neighbor look register closest neighbor by thinking about separation as highlight. In this unique circumstance, closest neighbor seek concentrate on finding closest neighbors where watchwords and spatial information plays a noteworthy effect. It accompanies calculations to answer such question. (Measure 10 and Normal)This report is a format. $\mathrm{n}$ electronic duplicate can be downloaded from the meeting site. For inquiries on paper rules, please contact the meeting productions board as demonstrated on the gathering site. Data about definite paper accommodation is accessible from the gathering site.
Recognizing a best course in a street organize is a vital issue that discovers applications in outline. For instance, delineate like BaiduLvyou 1 and Yahoo Travel 2 offer apparatuses for trip arranging. In any case, the courses that they give are gathered from clients and are in this manner pre-characterized. This is a noteworthy lack since there may not exist any pre-characterized course that meets the client needs. The current answers for trip arranging or course seek are frequently lacking in offering the adaptability for clients to determine their prerequisites on the course.

Consider a client who needs to spend a day investigating a city. She isn't acquainted with the city and she may stance such a question: "Locate the most prominent course to and from my inn to such an extent that it goes by shopping center, eatery, and bar, and the time spent out and about altogether is inside 4 hours."

The case inquiry above has two hard requirements: 1) the purposes of interests favored by the client, as communicated by an arrangement of catchphrases that ought to be shrouded in the course (e.g., "shopping center", "eatery" and "bar"); 2) a spending limitation (e.g., travel time) that ought to be fulfilled by the course. The inquiry intends to recognize the ideal course under the two hard requirements, with the end goal that a target score is streamlined (e.g., course ubiquity [4]). Note that course prevalence can be evaluated by the quantity of clients voyaging a course, got from the client voyaging histories recorded in sources, for example, GPS directions or Flickr photographs [4]. When all is said in done, the spending limitation and the target score can be of different 
distinctive composes, for example, travel span, remove, prominence, travel spending plan, and so forth. We consider two distinct qualities for spending imperative and target score since clients regularly need to adjust the exchange off of two viewpoints when arranging their treks. For instance, a prominent course might be very costly, or a course with the briefest length is of little interests. In the case question, it is likely that the most prominent course requires voyaging time over 4 hours. Henceforth, a course seeking framework ought to have the capacity to adjust such exchange offs as indicated by clients' distinctive inclinations.

We allude to the previously mentioned sort of questions as catchphrase mindful ideal course inquiry, indicated as KOR. Formally, a KOR question is characterized over a chart G, and the contribution to the inquiry comprises of five parameters, versus, vt, $\psi, \Delta$, and $f$, where versus is the source area of the course in G, vt is the objective area, $\psi$ is an arrangement of catchphrases, $\Delta$ is a spending farthest point, and $\mathrm{f}$ is a capacity that ascertains the target score of a course. The question restores a way $\mathrm{R}$ in $\mathrm{G}$ beginning at versus and finishing at vt, with the end goal that $R$ limits $f(R)$ under the imperatives that $\mathrm{R}$ fulfills as far as possible $\Delta$ and goes through areas that cover the inquiry catchphrases in $\psi$. To the best of our insight, none of the current work on trip arranging or course look is appropriate for KOR inquiries. Moreover, the issue of unraveling KOR questions can be appeared to be NP-hard by a decrease from the weighted compelled briefest way issue [8]. It can likewise be seen as a summed up voyaging sales representative issue [11] with limitations. This prompts an intriguing inquiry: is it conceivable to infer proficient answers for noting KOR questions.

Because of the hardness of noting KOR inquiries, in this paper, we answer the previously mentioned inquiry positively with three guess calculations. The principal estimation calculation has an execution bound and is indicated by OSScaling. In OSScaling, we first scale the target estimation of each edge to a whole number by a parameter $o$ to acquire a scaled diagram indicated by GS. In particular, in the scaled diagram GS, every incomplete course is spoken to by a "mark", which records the question catchphrases officially secured by the halfway course, the scaled target score, the first target score, and the spending score of the course. At every hub, we keep up a rundown of "helpful" names comparing to the courses that go to that hub. Beginning from the source hub, we continue making new halfway courses by broadening the present "best" fractional course to produce new marks, until the point when all the possibly helpful names on the objective hub are created. At long last, the course spoke to by the name with the best target score at the objective hub is returned.

\section{Related Work}

The substance utilized for questioning appears as spatial database Best catchphrase cover inquiry takes type of watchwords or items. For instance: school. Given a spatial database P, comprise of set of focuses. For a question q, where q have a place with set of items, it look for closest neighbor inside the protest via looking through its or better basic leadership, idea of catchphrase rating was presented alongside its highlights other than separate. For such inquiry, question will take type of highlight of articles. It scan for closest neighbor in light of another comparability measure, named weighted normal of list rating which join watchword rating, catchphrase look and closest neighbor seek. Gauge calculation requires spatial protests as records which incorporate fields like spatial area and its report identifier and its address. Spatial items are objects gotten from spatial information. All operations rotate around spatial items. Contribution to gauge calculation requires single inquiry catchphrase as items. The initial phase in pattern calculation is to set a variable bkc as zero. The subsequent stage is to produce applicant watchword cover. Applicant catchphrase cover produce spatial articles that contain those inquiry watchwords. Watchword centrality has been figured utilizing term recurrence backwards record recurrence as likeness measure. Term recurrence reverse report recurrence is a mix of term recurrence and backwards record recurrence.

The default esteem is set as zero. The score acquired is contrasted and first score. In the event that its esteem is more noteworthy than zero, it has been set as best watchword cover. Score figuring can be acquired as a pruning procedure. The subsequent stage is to perform closest neighbor look upon applicant watchword covers created. Closest neighbor look calculation has been processed utilizing a customary closeness measure named Euclidean separation. This similitude measure depends on separate. Closest neighbor seek calculation sets its default an incentive regarding clients current client area. In light of that area, rest of separation as for that area has been ascertained. The one slightest separation as for inquiry area has been viewed as best catchphrase cover. At the point when number of question watchwords expands, its execution drops. It running time is high.

\section{Literature Survey}

Ke Deng [1] accompanies calculations to discover closest neighbor utilizing catchphrases. Joao B Rocha [2] proposed spatial reversed record, a variation of altered file to store watchwords. Xin Cao [3] proposed the idea of aggregate spatial watchword questioning. The focal thought is to look for aggregate protests that all things considered fulfill an inquiry. Closest neighbor look likewise goes under classification of seeking process. In view of this idea, Gisli R [4] proposed remove perusing calculation in spatial databases. Ronald Fagin [5] managed ideal conglomeration calculation which helps in quick watchword seek.

Yufei Tao [6] proposed strategy for finding closest neighbors utilizing tree structure as list. Lisi Chen [7] give an overview of files to store catchphrases and spatial area. Xin Cao [8] managed different spatial watchword questions. The idea of Boolean range question fall under the class of spatial catchphrase inquiry. Dong Xiang Zhang [9] proposed adaptable coordinated altered record for putting away spatial information. Bolin Ding [10] gives technique to effectively process watchword inquiries. Shuyao [11] proposed the idea of watchword inquiry. Jianhua [12] considered a type of record named watchword match based structure for discovering top $\mathrm{k}$ answers utilizing catchphrase seek.

Travel course look: The movement course seek issue has gotten a great deal of consideration. Li et al. [17] propose another inquiry called Trip Planning Query (TPQ) in spatial databases, in which each spatial protest has an area and a classification, and the articles are recorded by a R-tree. A TPQ has three parts: a begin area $\mathrm{s}$, an end area $\mathrm{t}$, and an arrangement of classifications $\mathrm{C}$, and it is to locate the most limited course that begins at $\mathrm{s}$, goes through no less than one question from every classification in $\mathrm{C}$ and closures at $t$. It is demonstrated that TPQ can be lessened from the Traveling Salesman issue, which is NP-hard. In view of the triangle imbalance property of metric space, two guess calculations including a ravenous calculation and a whole number programming calculation are proposed. Contrasted and TPQ, KOR contemplated in this paper incorporates an extra requirement (the spending imperative), and hence is more expressive. The calculations in the work [17] can't be utilized to process KOR.

Sharifzadeh et al. [22] examine a variation issue of TPQ [17], called ideal sequenced course question (OSR). In OSR, an aggregate request on the classes $\mathrm{C}$ is forced and just the beginning area $\mathrm{s}$ is determined. The creators propose two rich correct calculations LLORD and R-LORD. Under a similar setting [17] that articles are put away in spatial databases and listed by a R-tree, metric 
space based pruning systems are produced in the two correct calculations. Chen et al. [3] considers the multi-manage halfway sequenced course (MRPSR) inquiry, which is a brought together question of TPQ and OSR. Three heuristic calculations are proposed to answer MRPSR. KOR is not quite the same as OSR and MRPSR and the their calculations are not material to process KOR.

Kanza et al. [14] consider an alternate course seek inquiry on the spatial database: the length of the course ought to be littler than a predetermined limit while the aggregate content significance of this course is boosted. Voracious calculation is proposed without ensuring to locate an achievable course. Their ensuing work [12] builds up a few heuristic calculations for noting a comparative question in an intuitive way. Subsequent to going to each protest, the client gives criticism on whether the question fulfills the in quiry, and the input is considered when registering the following item to be gone to. In the work [16], surmised calculations for comprehending OSR [22] within the sight of request imperatives in an intelligent way are created. Kanza et al. likewise contemplate the issue of looking ideal sequenced course in probabilistic spatial database [13]. Lu et al. [18] consider a similar question [14] and propose an information mining-based approach. The questions considered in these works are not the same as KOR and these calculations can't be utilized to answer KOR.

Malviya et al. [20] handle the issue of noting consistent course arranging questions over a street organize. The course arranging [20] intends to locate the most brief way within the sight of updates to the postpone gauges. Roy et al. [21] consider the issue of intelligent excursion arranging, in which the clients give criticisms for the as of now recommended purposes of-interests, and the agendas are built iteratively in light of the clients' inclinations and time spending plan. Clearly, these two issues are diverse with KOR.

Yao et al. [23] propose the multi-inexact watchword directing (MARK) inquiry. A MARK inquiry is determined by a beginning and a closure area, and an arrangement of (catchphrase, limit) esteem sets. It looks for the course with the most brief length to such an extent that it covers no less than one coordinating item for each catchphrase with the closeness bigger than the relating limit esteem. Clearly, MARK has distinctive points with that of the KOR question.

The aggregate spatial catchphrase seek [2] is identified with our concern, where a gathering of articles that are near a question point and on the whole cover an arrangement of an arrangement of inquiry watchwords are returned as the outcome. Notwithstanding, the KOR question requires a course fulfilling a spending imperative as opposed to an arrangement of free areas. Our concern is additionally important to the spatial catchphrase look inquiries [1, 6] where both spatial and literary highlights are considered amid the question handling. In any case, they recover single articles while the KOR question finds a course.

Travel course suggestion: Recent takes a shot at set out course proposal intend to prescribe courses to clients in light of clients movement histories. Lu et al. [19] gather geo-labeled photographs from Flickr and assemble travel courses from them. They characterize prominence scores on every area and each trek, and prescribe a course that has the biggest prevalence score inside a movement length in the entire dataset for a city. The suggestion in this work isn't planned as inquiries and the proposal calculation keeps running in an outrageous in length time. The work [4] finds mainstream courses from clients' chronicled directions. The fame score is characterized as the likelihood from the source area to the objective area assessed utilizing the retaining Markov demonstrate in light of the directions. Yoon et al. [24] propose a savvy suggestion, in light of different client produced GPS directions, to pro- ductively discover schedules. The work [15] predicts the consequent courses as per the client's present direction and past direction history. None of these recommendations considers the watchwords as we do in this work.

\section{Proposed System}

Since all execution operations rely upon objects, there exist an issue of picking which protests first to query when given various highlights of various articles. For this reason watchword rating has been related with objects. Rating is based everyday significance of protest in day by day life. Rating takes estimation of whole number going from 1 to 5 . This calculation include watchword rating as well as include highlights of articles also. Articles must be chosen to include highlights.

Contribution to catchphrase closest neighbor extension variation calculation is an arrangement of inquiry watchwords as highlights related with objects. The initial step is to choose rule inquiry watchword to perform seek. At the end of the day, to distinguish the principal protest in which highlight has been related for seeking. Items connected with guideline question watchword are called rule objects. Ordering has been utilized to discover required protest related with watchword. In the wake of recognizing the protest, it scan for objects having most elevated catchphrase rating. The one with most elevated watchword rating are normally set as the principal protest in which seek must be done.

It additionally discovers best course seek. In the event that element is set as info, initial step is to distinguish the question with most noteworthy watchword rating to perform seek. At that point closest neighbor look calculation has been performed to discover closest neighbor of client's question as for current area. From current protest, next question with most noteworthy catchphrase rating has been recognized. At that point is include as for second protest has been computed and esteem is gotten. Along these lines best course look has been acquired. This paper likewise finds Boolean range question utilizing catchphrase look. Question takes type of watchword and its range. Question closest to that range will be shown therefore.

\subsection{Baseline Algorithm}

With increment in watchwords for catchphrase seek and closest neighbor look, there is a need to perform inquiry on such information. Pattern calculation concentrate on recovering information concerning question catchphrases. Best watchword cover can be gotten by pattern calculation.

Pattern calculation requires spatial questions as records which incorporate fields like spatial area and its report identifier and its address. Spatial items are objects gotten from spatial information. All operations spin around spatial items. Contribution to benchmark calculation require single question catchphrase as items. The initial phase in gauge calculation is to set a variable bkc as zero. The subsequent stage is to produce applicant catchphrase cover. Hopeful watchword cover create spatial items that contain those inquiry catchphrases. Watchword importance has been ascertained utilizing term recurrence reverse archive recurrence as likeness measure. Term recurrence backwards report recurrence is a mix of term recurrence and converse record recurrence. Term recurrence is the circumstances a term happen in a solitary record separated by add up to number of terms in a report. Reverse report recurrence is the

converse of number of times a term happens in an archive separated by add up to number of records. Term recurrence opposite report recurrence is the result of term recurrence and reverse archive recurrence. 
The default esteem is set as zero. The score acquired is contrasted and first score. In the event that its esteem is more noteworthy than zero, it has been set as best watchword cover. Score count can be acquired as a pruning methodology. The following stage is to perform closest neighbor seek upon hopeful catchphrase covers created. Closest neighbor seek calculation has been figured utilizing a customary likeness measure named Euclidean separation. This likeness measure depends on remove. Closest neighbor look calculation sets its default an incentive as far as clients current client area. In light of that area, rest of separation as for that area has been ascertained. The one slightest separation as for inquiry area has been viewed as best catchphrase cover. At the point when number of inquiry watchwords builds, its execution drops. It running time is high.

\subsection{Keyword Nearest Neighbor Expansion Variant Algorithm}

Gauge calculation just recover reply to single inquiry. It doesn't react to various inquiry watchwords. It doesn't react to spatial highlights. To defeat the downside, the creator proposed a variation of closest neighbor calculation called watchword closest neighbor extension variation. This calculation concentrate on recovering closest neighbor by consolidating both catchphrase seek and closest neighbor look. The new calculation present the idea of catchphrase rating, spatial importance and watchword significance. Watchword rating helps in basic leadership. For better basic leadership catchphrase rating assume a huge part.

Since all execution operations rely upon objects, there exist an issue of picking which protests first to query when given various highlights of various articles. For this reason catchphrase rating has been related with objects. Rating is based everyday significance of question in day by day life. Rating takes estimation of whole number running from 1 to 5 . This calculation include catchphrase rating as well as include highlights of items also. Items must be chosen to include highlights.

Contribution to watchword closest neighbor extension variation calculation is an arrangement of question catchphrases as highlights related with objects. The initial step is to choose standard question watchword to perform seek. As it were, to distinguish the principal protest in which include has been related for looking. Items connected with guideline inquiry catchphrase are called rule objects. Ordering has been utilized to discover required protest related with watchword. In the wake of recognizing the question, it look for objects having most elevated watchword rating. The one with most astounding catchphrase rating are generally set as the main protest in which seek must be completed.

For each sub objects, score has been figured. Sub protests alongside its score is called nearby best catchphrase cover. Neighborhood best catchphrase cover has been gotten with the assistance of likeness measure named weighted normal of record rating. Weighted normal of list rating consolidates both catchphrase look and closest neighbor seek. When nearby best catchphrase cover has been processed, the one with most elevated score comes about as the best watchword cover. Score computation acquires redress information. All together for clients seeing, closest neighbor seek calculation has been called.

It additionally discovers best course look. On the off chance that element is set as information, initial step is to distinguish the question with most astounding watchword rating to perform look. At that point closest neighbor seek calculation has been performed to discover closest neighbor of client's question as for current area From current question, next protest with most noteworthy catchphrase rating has been recognized. At that point is include concerning second protest has been figured and esteem is gotten.
Consequently best course look has been acquired. This paper additionally finds Boolean range question utilizing catchphrase seek. Inquiry takes type of watchword and its range. Question closest to that range will be shown therefore.

\section{Analysis}

The proposed technique accompanies two calculations. Our trial depends on genuine information. The dimensionality is dependably 2 . Pattern calculation connected on genuine information concentrate on recovering information utilizing single question watchword. Catchphrase closest neighbor development variation calculation recover information utilizing various inquiry watchword. Fig 1 demonstrates bar chart speaking to execution time of standard calculation versus dataset check or number of records scanned for a specific inquiry watchword. Vertical pivot demonstrate execution time amid seek process. Execution time is the contrast between begin time and run time when seek technique happen. At the point when a solitary question catchphrase is looked in a record of thirty four, its execution time is 8433 milliseconds. Additionally, while seeking takes in a document tally of three for one question watchword, its execution time is 508 milliseconds. While looking happens in a document tally of twenty seven, execution time is 5071 milliseconds. It has been watched that execution time increments as records to be sought increments. Record check is directly corresponding to execution time. catchphrase cover tally of watchword closest neighbor extension variation calculation. At the point when catchphrase cover check is one, its execution time is 82 milliseconds. At the point when catchphrase cover check is two, its execution time is 121 milliseconds. At the point when catchphrase cover tally is three, its execution time is 127 milliseconds.

With the ubiquity of web-based social networking (e.g., Facebook and Flicker), clients can without much of a stretch offer their registration records and photographs amid their outings. In perspective of the colossal number of client verifiable portability records in online networking, we expect to find head out encounters to encourage trip arranging. When arranging an excursion, clients dependably have particular inclinations with respect to their outings. Rather than confining clients to constrained inquiry alternatives, for example, areas, exercises, or eras, we consider subjective content portrayals as watchwords about customized prerequisites. Additionally, an assorted and agent set of suggested travel courses is required. Earlier works have expounded on mining and positioning existing courses from registration information. To address the issue for programmed trip association, we assert that more highlights of Places of Interest (POIs) ought to be removed. In this manner, in this paper, we propose a productive Keyword-mindful Representative Travel Route structure that utilizations information extraction

from clients authentic versatility records and social co-operations. Expressly, we have composed a watchword extraction module to characterize the POI-related labels, for compelling coordinating with inquiry catchphrases. We have additionally planned a course recreation calculation to develop course competitors that satisfy the prerequisites. To give befitting inquiry comes about, we investigate Representative Skyline ideas, that is, the Skyline courses which best portray the exchange offs among various POI highlights. To assess the viability and productivity of the proposed calculations, we have directed broad analyses on genuine area based informal community datasets, and the investigation comes about demonstrate that our strategies do in reality exhibit great execution contrasted with best in class works. 


\section{Conclusion}

This paper tended to the topic of genuine travel course suggestion. The client input their tourism necessities, at that point the framework make great utilization of POI data to get the prominence and geo-directions of them. A point by point report of two calculations to recover best watchword cover was exhibited. Best watchword cover question expects to recoup spatial articles regarding client's prerequisite. Calculations are utilized to discover reply to such question. It likewise accompanies best watchword cover course look which discovers best course.

\section{References}

[1] Ke Deng, Xin Li and Xiao fang Zhou, "Best Keyword Cover Search", IEEE Transaction on Knowledge and Data Engineering, vol 27, no 1, January 2015.

[2] Joao B Junior, Orestis sGkorghas, Simon Jonassen and KjetilNorvag, “ Efficient Processing of Top K Spatial Key-word Queries", in SSTD, pages 205-222, 2012.

[3] Xin Cao, Gao Cong, Beng Chin, "Collective Spatial Key-word Querying”, ACM Transaction on Database Systems, 2011.

[4] Gilsi R Hjaltson and HansebSamet, "Distance Browsing in Spatial Databases", ACM Transaction on Database Systems, June 1999, pp 265-318.

[5] Ronald Fagin, Ammon Lotem and MoniNaor, "Optimal Aggregation Algorithms for Middleware", Journal of Com-puter and System Sciences, April 2003.

[6] Yufei Tao and Cheng Sheng, "Fast Nearest Neighbor Search with Keywords", IEEE Transaction on Knowledge and Data Engineering, vol 26, no 4, April 2014.

[7] Lisi Chen, Geo Cong, Christian S Jensen, "Spatial Key-word Query Processing: An experimental evaluation", Pro-ceedings of the VLDB Endowment, vol 6, no 3, 2013.

[8] X. Cao, L Chen, Gao Cong, C. S Jensen, "Spatial Key-word Querying", Information Forensics and Security, Spring-er 2012.

[9] Dong Xiang Zhang, Kian Lee Tan, Anthony K. H. Tung," "Scalable Top K Spatial Keyword Search”, ICDT , March 2015.

[10] J.M. Kleinberg. Authoritative sources in a hyperlinked environment Journal of the ACM (JACM), 46(5):604-632, 1999.

[11] K. Kodama, Y. Iijima, X. Guo, and Y. Ishikawa. Skyline queries based on user locations and preferences for making lo-cation-based recommendations. In LBSN, pages 9-16. ACM, 2009.

[12] J.A. Konstan, B.N. Miller, D. Maltz, J.L. Herlocker, L.R. Gordon, and J. Riedl.Grouplens: applying collaborative filter-ing to use net news. Communications of the ACM, 40(3):77-87, 1997.

[13] C.H. Lee, Y.H. Kim, and P.K. Rhee. Web personalization expert with combining collaborative filtering and association rule mining technique. Expert Systems with Applications, 21(3):131-137, 2001.

[14] J. Levandoski, Mohamed Sarwat, Ahmed Eldawy, and Mohamed Mokbel. Lars: A location-aware recommender sys-tem. In ICDE, 2012.

[15] H. Ma, H. Yang, M.R. Lyu, and I. King.Sorec: social recommendation using probabilistic matrix factorization. In CIKM, pages 931940. ACM, 2008.

[16] Mohamed Mokbel, JieBao, Ahmed Eldawy, Justin Levandoski, and Mohamed Sarwat. Personalization, Socializa-tion, and Recommendations in Location-based Services 2.0.In PersDB. VLDB, 2011.

[17] Noulas, S. Scellato, C. Mascolo, and M. Pontil. Ex-ploiting semantic annotations for clustering geographic areas and users in location-based social networks. In Fifth Interna-tional AAAI Conference on Weblogs and Social Media, 2011.

[18] M.H. Park, J.H. Hong, and S.B. Cho. Location-based recommendation system using bayesian user's preference model in mobile devices. Ubiquitous Intelligence and Compu-ting, pages 1130-1139, 2007.

[19] S. Robertson. Understanding inverse document frequency: on theoretical arguments for idf. Journal of Documentation, 60(5):503-520, 2004

[20] S. Scellato, A. Noulas, R. Lambiotte, and C. Mascolo. Socio-spatial properties of online location-based social net-works. Proceedings of ICWSM, 11, 2011

[21] A.I. Schein, A. Popescul, L.H. Ungar, and D.M. Pen-nock. Methods and metrics for cold-start recommendations. In SIGIR, pages 253 260. ACM, 2002.
[22] M. Ye, P. Yin, and W.C. Lee. Location recommendation for location-based social networks. In SIGSPATAIL, pages 458-461. ACM 2010.

[23] Y. Zheng and X. Xie. Learning travel recommendations from usergenerated gps traces. ACM TIST, 2(1):2, 2011.

[24] Y. Zheng, X. Xie, and W.Y. Ma.Geolife: A collaborative social networking service among user, location and trajectory. IEEE Data Engineering Bulletin, 33(2):32-40, 2010. 